\title{
(6) OPEN ACCESS \\ Man or machine? An experimental study of prehospital emergency amputation
}

\author{
Caroline Leech, ${ }^{1}$ Keith Porter ${ }^{2}$
}

${ }^{1}$ Emergency Department, University Hospitals Coventry \& Warwickshire NHS Trust, Coventry, UK

${ }^{2}$ Academic Department of Clinical Traumatology, University of Birmingham, Birmingham, UK

\section{Correspondence to} Dr Caroline Leech, Emergency Department, University Hospitals Coventry \& Warwickshire NHS Trust, Clifford Bridge Road, Coventry, CV2 2DX, UK;

Caroline.leech@uhcw.nhs.uk

Received 29 March 2015 Revised 5 October 2015

Accepted 27 March 2016 Published Online First 8 June 2016
CrossMark

To cite: Leech C, Porter $\mathrm{K}$. Emerg Med J 2016;33: 641-644.

\section{ABSTRACT}

Objective Prehospital emergency amputation is a rare procedure, which may be necessary to free a time-critical patient from entrapment. This study aimed to evaluate four techniques of cadaveric lower limb prehospital emergency amputation.

Method A guillotine amputation of the distal femur was undertaken in fresh frozen self-donated cadavers. A prehospital doctor conducted a surgical amputation with Gigli saw or hacksaw for bone cuts and firefighters carried out the procedure using the reciprocating saw and Holmatro device. The primary outcome measures were time to full amputation and the number of attempts required. The secondary outcomes were observed quality of skin cut, soft tissue cut and CT assessment of the proximal bone. Observers also noted the potential risks to the rescuer or patient during the procedure.

Results All techniques completed amputation within $91 \mathrm{~s}$. The reciprocating saw was the quickest technique (22 s) but there was significant blood spattering and continuation of the cut to the surface under the leg. The Holmatro device took less than a minute. The quality of the proximal femur was acceptable with all methods, but $5 \mathrm{~cm}$ more proximal soft tissue damage was made by the Holmatro device.

Conclusions Emergency prehospital guillotine amputation of the distal femur can effectively be performed using scalpel and paramedic shears with bone cuts by the Gigli saw or fire service hacksaw. The reciprocating saw could be used to cut bone if no other equipment was available but carried some risks. The Holmatro cutting device is a viable option for a life-threatening entrapment where only firefighters can safely access the patient, but would not be a recommended primary technique for medical staff.

\section{INTRODUCTION}

Emergency amputation is a very rare but potentially life-saving intervention in the prehospital setting. It may be required due to entrapment in the wreckage of a road traffic collision, agricultural or industrial machinery.

The indications to perform prehospital amputation include:

1. an immediate and real risk to the patient's life due to a scene safety emergency

2. a deteriorating patient physically trapped by a limb when they will almost certainly die during the time taken to secure extrication

3. a completely mutilated non-survivable limb retaining minimal attachment, which is delaying extrication and evacuation from the scene in a non-immediate life-threatening situation

\section{Key messages}

What is already known on this subject? Prehospital emergency amputation is a rare but potentially life-saving procedure. There is little evidence about which technique or equipment may be effective. This cadaveric study aimed to assess four different techniques of amputation using equipment, which may be carried by a prehospital doctor and the UK fire service.

\section{What might this study add?}

The fire service hacksaw could be used if a Gigli saw was not available to cut bone. The Holmatro dedicated cutter is a viable option for a life-threatening entrapment where only firefighters can safely access the patient.

4. the patient is dead and their limbs are blocking access to potentially live casualties. ${ }^{1}$

The majority of prehospital doctors in the UK have not been formally or practically trained to undertake emergency prehospital amputation although most organisations have standard operating procedures in place for this eventuality. A Gigli saw is most commonly used for an emergency prehospital amputation but this is not carried by every prehospital practitioner (personal communication).

The role of hydraulic equipment carried by the UK fire service has been considered as a potential last resort method for entrapped peri-arrest patients.

Review of the literature identified one study which assessed the role of a guillotine surgical amputation with a bone saw versus the Holmatro jaws of life at different levels using porcine and human cadaveric lower limbs. ${ }^{2}$

The aim of this study was to observe the results of lower limb amputation using a surgical technique with a Gigli saw; a surgical technique with a hacksaw; immediate amputation with a reciprocating saw and immediate amputation with a Holmatro device.

\section{METHODS}

The study was carried out in the West Midlands Surgical Training Centre, located at University Hospitals Coventry \& Warwickshire National Health Service (NHS) Trust, which is licensed by the Human Tissue Authority (license 30019).

Ethical approval was not required as research involving previously collected, non-identifiable tissue samples in accordance with the terms of 
donor consent is excluded from NHS Research Ethics Approval review. ${ }^{3}$ Permission was obtained from West Midlands Fire Service to use hydraulic equipment and a full risk assessment was conducted prior to the study. Personal protective equipment, including helmet with visor, face mask and heavy-duty gloves, was worn by the firefighter operators and a $3 \mathrm{~m}$ boundary was established for the observers.

Two fresh frozen self-donated cadavers with no preservative or arterial treatment, and of similar weight and size, were selected for the study. A point on the distal femur $10 \mathrm{~cm}$ above the superior border of the patella was measured and marked on each of the four limbs. This level was selected as an appropriate site to simulate a patient severely trapped by their lower limbs in a motor vehicle. The circumference of the thighs at this point ranged between 43.1 and $45.0 \mathrm{~cm}$ in the four lower limbs.

A Combat Application Tourniquet (C-A-T) was applied, proximal to the marked site, and tightened firmly with the windlass to simulate application for haemorrhage control.

Four amputation techniques were compared in this study. Two surgical procedures of guillotine amputation were performed by a single trauma surgeon with experience of emergency amputation in the field. These were: (1) using scalpel to cut the skin and fascia, paramedic shears to divide the muscles and Gigli saw to cut the femoral bone (figure 1), followed by paramedic shears to cut the remaining posterior soft tissue and (2) using scalpel, paramedic shears and a hacksaw from the fire service equipment (figure 2) with a similar technique to above. The surgical operator was assisted by a critical care paramedic (not previously involved in an amputation) who passed equipment and protected sharps.

A further two procedures were performed by the fire service personnel direct to skin using the Dewalt reciprocating saw (figure 3) and the Holmatro dedicated cutter (figure 4): both are routinely carried on local fire service appliances. The ranks of the fire service operators were watch commander and crew commander, respectively, and both had significant experience of using the equipment in operational duties. The firefighters were briefed by the first author just before the procedure, but no advice was provided during the timed experiment. Immediately after the procedure and 1 month later, the firefighters were contacted to ensure there were no adverse psychological reactions to being involved in the study.

The Dewalt DC305 is a cordless handheld $3.7 \mathrm{~kg}$ reciprocating saw with a $36 \mathrm{~V}$ rechargeable battery. The normal use is for

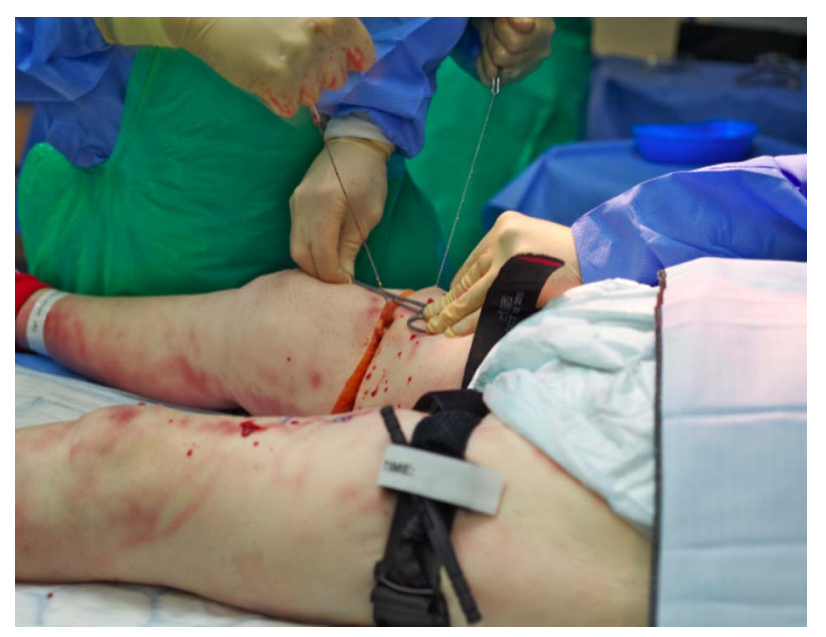

Figure 1 Guillotine amputation with Gigli saw.

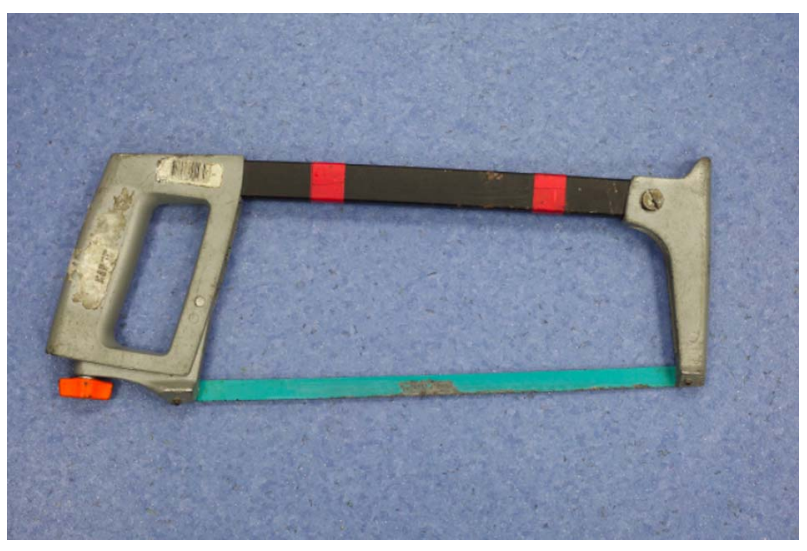

Figure 2 Fire service hacksaw.

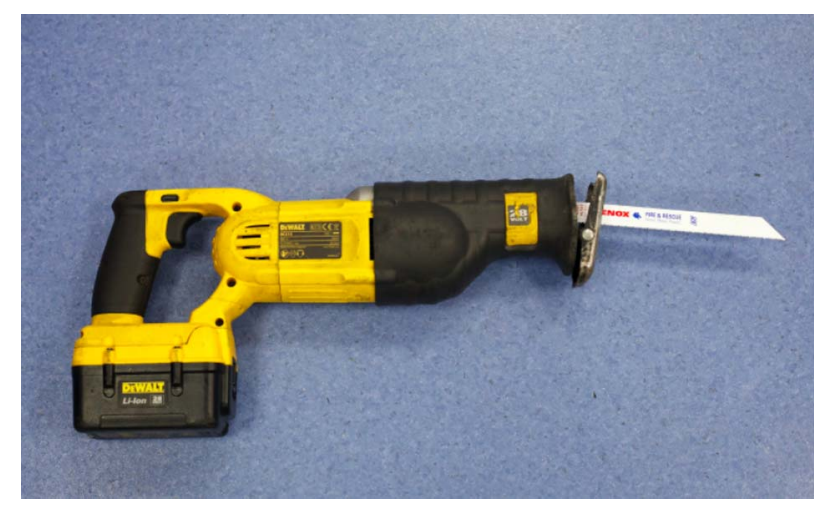

Figure 3 Reciprocating saw.

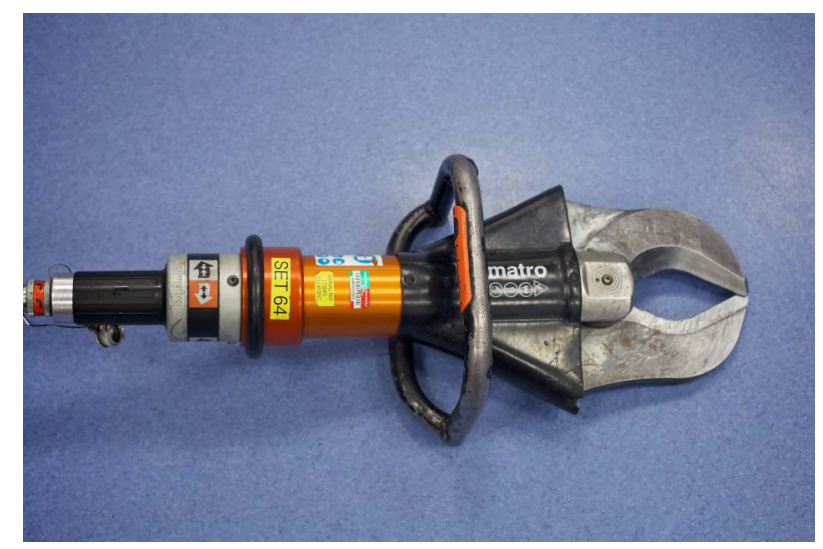

Figure 4 Holmatro dedicated cutter.

cutting wood, plastic or metal. It has an adjustable saw shoe at the base of the saw, which leaves an exposed blade length of $200 \mathrm{~mm}$, and the stroke length is $28.5 \mathrm{~mm}$. Dust respirators must be worn during use due to the risk of aerosolation. The device used had been fully charged but did not have a new blade fitted and had previously been used for cutting motor vehicles in extrication training.

The Holmatro dedicated cutter CU 3020 has specially developed blades, which are normally used for cutting vehicle roof, door and window posts. The cutting force in the centre of the blades is $188 \mathrm{kN}$ (19 tonnes). The weight of the device is $12.5 \mathrm{~kg}$ and the blades open to an internal distance of $159 \mathrm{~mm}$ or an external width of $256 \mathrm{~mm}$. The rescue tool operates on a 
'Deadman's Handle' principle, which means when the operating handle is released, the blades return to the neutral position and tool movement ceases. The Holmatro dedicated cutter can be used under water and down to temperatures of $-20^{\circ} \mathrm{C}$. The device is normally connected via a dual core single hose line to a hydraulic pump running with unleaded fuel but for the purposes of the indoor experiment an electric pump was used to avoid fumes.

The primary outcome measures were the time from ' $\mathrm{knife}$ to skin' to full amputation with the limb disconnected; the number of attempts and the perceived risks to the rescuer or patient during the procedure. Postprocedure, the secondary outcomes of quality of the skin, soft tissue and bone cut were assessed by inspection of the proximal stump. No credible grading system was found to score the quality of cut so a system of grading was devised (grade $1=$ very poor quality, very ragged cut, unsalvageable damage; 2 =poor quality, ragged cut, significant damage; $3=$ average quality, acceptable damage; $4=$ good quality, moderately smooth cut, minimal damage and grade 5 was excellent quality, smooth cut, no obvious damage). Six clinical raters independently analysed the procedure and observed the quality of the incisions immediately after the procedure. The raters were not blinded to the technique used and were experienced consultants from a prehospital background. The surgical operator did not rate their own amputations. The mean scores are presented in the results. The operators of each procedure also gave qualitative feedback.

This experiment was conducted during the West Midlands Pre-Hospital Emergency Surgical Skills Course. Amputations were repeated, with the Gigli saw and hacksaw, by 14 other prehospital (non-surgical) doctors on the course but not graded.

CT scans of the proximal stump were performed the same day as the procedure. The scans were reported by an experienced consultant radiologist who specialises in musculoskeletal trauma; who did not see the cadaveric specimens and was blinded to the cutting technique.

\section{RESULTS}

The results of the four amputation techniques can be found in table 1.

The time to set up the equipment was not formally recorded but was negligible with all methods.

Amputation was completed by all techniques in less than $91 \mathrm{~s}$. The hacksaw jammed on the bone and required resiting, which accounted for the three cuts. Two different angles were required by the reciprocating saw to complete the amputation. With the Holmatro device, two cuts were required, allowing time for the jaws to open and close again, and a small amount of posterior bridging soft tissue remained, which was cut by the firefighters using paramedic shears.

CT images of the four limbs revealed that all bone cuts were satisfactory. There was no fracture propagation of the proximal bone or comminution of the cut bone with any technique. The quality of the Holmatro bone cut on CT appeared to be superior to the grading given by the observers. The bone cut was more angled with the Gigli saw and transverse with the hacksaw surgical technique. From the reported CT images, there was $5 \mathrm{~cm}$ more proximal soft tissue injury using the Holmatro technique.

The firefighters reported that there was no feedback, vibration or resistance when operating the Holmatro or Dewalt reciprocating saw. There were no reported adverse psychological effects.

\section{DISCUSSION}

This is the first published study to explore the feasibility of more than one method of emergency prehospital lower limb amputation using fire service equipment. The results found that all methods of emergency amputation were viable and could be executed within $91 \mathrm{~s}$ in study conditions. Where a skilled prehospital doctor can access the patient, the technique of using a scalpel, paramedic shears and then either a Gigli saw or a fire service hacksaw are both effective rapid methods producing a good quality proximal stump.

If only a firefighter could access the patient due to hazards (car on fire, rising flood water or chemical contamination), the reciprocating saw direct to skin was the most rapid technique but carried significant risks to the rescuer of blood splattering, aerosolation of tissue and bone and cutting through the surface under the limb. There was no obvious significant vibration transmitted from the reciprocating saw and the cut was effortless. The firefighters reported that they would have concerns that the battery might fail during the procedure and that the technique involved a definitive action by the firefighter. However, they felt that this was the quickest technique and therefore preferred for a very extreme situation such as a vehicle on fire. The Dewalt cordless saw is a smaller device and therefore may be easier to use in a confined space with no trailing cable within the cordon.

If no other equipment was available for cutting through the bone, a viable solution would be for a doctor to use scalpel and

Table 1 Results of amputation techniques

\begin{tabular}{|c|c|c|c|c|}
\hline & Gigli saw & Hacksaw & Reciprocating saw & Holmatro \\
\hline Time (s) & 91 & 88 & 22 & $38+15$ \\
\hline Number of cuts & 1 & 3 & 2 & $2+$ \\
\hline $\begin{array}{l}\text { Risks to rescuer or } \\
\text { survivor }\end{array}$ & $\begin{array}{l}\text { Sharp knife, sharp } \\
\text { Gigli saw }\end{array}$ & Sharp knife, saw blade injury & $\begin{array}{l}\text { Significant spray/splattering of blood and } \\
\text { tissue } \\
\text { Aerosolation of tissue would require FFP3 } \\
\text { mask } \\
\text { No feedback when cut complete so went } \\
\text { through to inferior surface }\end{array}$ & $\begin{array}{l}\text { Manual handling. Audible loud } \\
\text { splintering sound }\end{array}$ \\
\hline $\begin{array}{l}\text { Practicalities of use in } \\
\text { entrapment }\end{array}$ & Elbow room for Gigli & $\begin{array}{l}\text { Need to support/traction leg. } \\
\text { Difficult to angle hacksaw }\end{array}$ & $\begin{array}{l}\text { Some angulation required. Difficult to see } \\
\text { when cut completed }\end{array}$ & $\begin{array}{l}\text { Would be difficult to encircle leg } \\
\text { if within vehicle }\end{array}$ \\
\hline Quality of skin cut & 5 & 5 & 5 & 4 \\
\hline Quality of soft tissue cut & 5 & 5 & 4.5 & 3 \\
\hline Quality of bone cut & 4 & 5 & 4.5 & 2.5 \\
\hline
\end{tabular}


paramedic shears for the skin and soft tissue and the reciprocating saw for the bone. A risk would be the continuation of the cut through the bone and into the soft tissue, but the CT results from this single example indicate that this will not necessarily impact on the proximal stump quality.

In the amputation with the Holmatro device, the operator had to wait for the jaws to open and close again to cut deeper tissue and then still had to use the paramedic shears to cut through the posterior soft tissue of the thigh, which delayed the amputation. It was expected that the Holmatro cutting device would fragment the bone and there was indeed a loud splintering sound using the jaws, which would be unpleasant for bystanders. However, there was no evidence of bone fragmentation of the proximal stump on CT. The Holmatro did produce the most soft tissue damage proximally for reasons which were unclear. However, $5 \mathrm{~cm}$ may not be significant, as a more proximal level will be made for all patients during definitive terminalisation of the stump in hospital. An added advantage of the Holmatro device is that it can work underwater so it may be a valid option for the trapped immersed patient where other surgical techniques are impossible.

There were several limitations to this study. The simulation was not true to life in respect of taking place in a training centre with adequate lighting; the cadavers were not trapped and positioned supine on a table and there were no other clinical demands or emotive element to the procedure. The access to a trapped casualty in a Road Traffic Collision (RTC) may be more complex, especially when attempting to angle the reciprocating saw or to place the blades of the Holmatro device around the femur. The surgical operator was highly skilled in amputation: this is not representative of the average consultant level prehospital doctor. When the procedure was repeated with the Gigli saw and hacksaw by non-surgical doctors on the course, it was found to be technically similar but took up to a minute longer. The Gigli saw was found to be most difficult to use by nonsurgical operators and the authors recommend that prehospital practitioners practise the technique before needing to use the skill. This could be done by using a Gigli saw to cut through a small fixed tree branch. The skill of the firefighters using the equipment for amputation is likely to be generalisable to other firefighters in the real-life setting.

The lower limbs were selected as being a maximum of 19 $\mathrm{mm}$ difference in circumference but were from two different cadaveric bodies so may have had different tissue and bone strengths. The cadaveric specimens were elderly and may have had osteoporotic bone compared with a younger trapped prehospital patient. The preservation method may have had an effect on the pliability and retractability of the muscle after cutting. There was a low resting blood volume in the cadaveric specimens: this meant that haemorrhage could not be reliably assessed during the techniques. Despite application of a proximal tourniquet, there was a very small amount of blood loss detected from all limbs and a significant amount of blood splatter from the reciprocating saw. In real-life, the practitioner may well apply two tourniquets proximally and this would be recommended for future studies.

Only one attempt was made with each of the four methods (due to the availability of cadaveric specimens) providing a very small sample size. The different techniques were only trialled on the distal femur: chosen as a likely site for the entrapped RTC patient. Other sites such as the knee joint, the tibia or the ankle could be explored in future studies.
This evaluation found different results compared with the above knee amputations in the previous published study. The McNicholas study ${ }^{2}$ used an orthopaedic bone saw which is not available in the prehospital environment; the Holmatro hydraulic device was similar. The surgical techniques used medial and lateral longitudinal incisions for soft tissue flaps: this would not be indicated in the emergency setting and explains why the time to amputation was slightly longer. The Holmatro above knee amputation in the McNicholas study took half the time of the cut in this study with the same number of cutting actions, and it is unclear why this might be. The results of above knee amputation with the Holmatro device found $40 \mathrm{~mm}$ of proximal fracture propagation on visual inspection: this was not seen in our study with the benefit of CT images.

The raters measuring the subjective outcomes worked individually without conferring, but had seen the technique performed, creating potential observer bias. The radiologist reporting the CT scans of the proximal femurs was blinded to the technique used and reported that the results were very obvious with low risk of interobserver variability.

This study has not considered how the fire service equipment would be decontaminated following emergency medical use on human tissue or the psychological impact of firefighters being involved in a surgical amputation.

\section{CONCLUSION}

Emergency prehospital guillotine amputation of the distal femur can effectively be performed using scalpel and paramedic shears with bone cuts by the Gigli saw or fire service hacksaw. The reciprocating saw could be used to cut bone if no other equipment was available but carries risks of blood splattering and deeper uncontrolled continuation of the cut. The Holmatro cutting device is a viable option for a life-threatening entrapment where only firefighters can safely access the patient, but would not be a recommended primary technique for medical staff.

Acknowledgements The authors would like to thank Brian Burnett and Mark Ferriby from the West Midlands Surgical Training Centre for their support hosting the study; Dr Michelle Christie-Large, Consultant Radiologist, for reporting the CT scans; West Midlands Fire Service for allowing the use of fire service equipment and Watch Commander Andrew Tite, Crew Commander Tom Hatfield, Crew Commander James Barlow and Crew Commander Michael Hawksby for participating in the study.

Contributors CL contributed to study conception and design, data collection and analysis and principal manuscript drafting. KP contributed to study design and manuscript appraisal/editing.

Competing interests $\mathrm{CL}$ is Director of the Pre-Hospital Emergency Medicine (PHEM) Emergency Surgical Skills Course, which covers prehospital emergency amputation. KP was the operator for the studied surgical technique. This study has been accepted as a poster at the Trauma Care 2015 conference.

Provenance and peer review Not commissioned; externally peer reviewed.

Open Access This is an Open Access article distributed in accordance with the Creative Commons Attribution Non Commercial (CC BY-NC 4.0) license, which permits others to distribute, remix, adapt, build upon this work non-commercially, and license their derivative works on different terms, provided the original work is properly cited and the use is non-commercial. See: http://creativecommons.org/ licenses/by-nc/4.0/

\section{REFERENCES}

1 Porter KM. Prehospital amputation. Emerg Med J 2010;27:940-2.

2 McNicholas MJ, Robinson SJ, Polyzois I, et al. 'Time critical' rapid amputation using fire service hydraulic cutting equipment. Injury 2011;42:1333-5.

3 Governance arrangements for research ethics committees: a harmonized edition. Department of Health, 2011. 